\title{
In vivo cartilage strain increases following medial meniscal tear and correlates with synovial fluid matrix metalloproteinase activity
} \author{
Amy L. McNulty ${ }^{a}$, Louis E. DeFrate ${ }^{a, *}$ \\ ${ }^{a}$ Department of Orthopaedic Surgery, Duke University Medical Center, Durham, NC, United States \\ ${ }^{\mathrm{b}}$ Department of Radiology, Duke University Medical Center, Durham, NC, United States
}

Teralyn E. Carter ${ }^{\mathrm{a}}$, Kevin A. Taylor ${ }^{\mathrm{a}}$, Charles E. Spritzer ${ }^{\mathrm{b}}$, Gangadhar M. Utturkar ${ }^{\mathrm{a}}$, Dean C. Taylor ${ }^{\mathrm{a}}$, Claude T. Moorman III ${ }^{\mathrm{a}}$, William E. Garrett ${ }^{\mathrm{a}}$, Farshid Guilak ${ }^{\mathrm{a}}$,

\section{A R T I C L E I N F O}

Article history:

Accepted 10 February 2015

\section{Keywords:}

MRI

Cartilage deformation

Mechanobiology

COMP

Aggrecan

Biomarker

\begin{abstract}
A B S T R A C T
Meniscal tears are common injuries, and while partial meniscectomy is a frequent treatment option, general meniscus loss is a risk factor for the development of osteoarthritis. The goal of this study was to measure the in vivo tibiofemoral cartilage contact patterns in patients with meniscus tears in relation to biomarkers of cartilage catabolism in the synovial fluid of these joints. A combination of magnetic resonance imaging and biplanar fluoroscopy was used to determine the in vivo motion and cartilage contact mechanics of the knee. Subjects with isolated medial meniscus tears were analyzed while performing a quasi-static lunge, and the contralateral uninjured knee was used as a control. Synovial fluid was collected from the injured knee and matrix metalloproteinase (MMP) activity, sulfated glycosaminoglycan, cartilage oligomeric matrix protein, prostaglandin $\mathrm{E}_{2}$, and the collagen type II cleavage biomarker C2C were measured. Contact strain in the medial compartment increased significantly in the injured knees compared to contralateral control knees. In the lateral compartment, the contact strain in the injured knee was significantly increased only at the maximum flexion angle $\left(105^{\circ}\right)$. The average cartilage strain at maximum flexion positively correlated with total MMP activity in the synovial fluid. These findings show that meniscal injury leads to loss of normal joint function and increased strain of the articular cartilage, which correlated to elevated total MMP activity in the synovial fluid. The increased strain and total MMP activity may reflect, or potentially contribute to, the early development of osteoarthritis that is observed following meniscal injury.
\end{abstract}

(c) 2015 Elsevier Ltd. All rights reserved.

\section{Introduction}

Meniscal tears are a common injury, with more than 850,000 meniscal surgeries performed each year in the United States and nearly twice as many worldwide (Arendt et al., 1999; Baker and Lubowitz, 2006). The menisci play a critical role in normal knee function by providing important load bearing capabilities, lubrication, proprioception, joint congruity, and joint stability (Ahmed and Burke, 1983; Haut Donahue et al., 2004; Markolf et al., 1981; Wojtys and Chan, 2005). Meniscal injury is associated with pain and degradative changes in the knee joint that may ultimately lead to osteoarthritis (Badlani et al., 2013; Berthiaume et al., 2005; Christoforakis et al., 2005; Hunter et al., 2006; Majewski et al., 2006; Roos et al., 1998;

\footnotetext{
* Correspondence to: Orthopaedic Research Laboratories, Department of Orthopaedic Surgery, Box 3093, Duke University Medical Center, Durham, NC 27710
} United States.
Sharma et al., 2008; Wyland et al., 2002). Furthermore, the surgical treatment of a meniscal tear by partial or total meniscectomy is strongly associated with articular cartilage degradation and the progression of osteoarthritis (Lohmander et al., 2007, 1994; Roos et al., 1998; Wyland et al., 2002). In fact, more than half of people with meniscectomy develop knee osteoarthritis within 5-15 years after joint injury (Lohmander et al., 2007, 1994). Therefore, surgeons attempt to preserve and repair the native meniscal tissue following injury (Abrams et al., 2013; Hutchinson et al., 2014; Lee et al., 2006; Maffulli et al., 2010). However, when repair is not feasible, partial meniscectomy is frequently implemented to treat meniscal tears. While these patients report improvements in pain and function, the ability of this surgery to mitigate the risk of premature development of osteoarthritis may be limited (Andersson-Molina et al., 2002; Fauno and Nielsen, 1992; Hall et al., 2014; Hoser et al., 2001; Katz et al., 2006; Rangger et al., 1995).

In order to better understand the mechanisms by which meniscal injury or meniscectomy predisposes the joint to 
osteoarthritis, changes in joint loading have been assessed using a variety of approaches, including finite element models (Peña et al., 2006; Peña et al., 2005; Wilson et al., 2003; Zielinska and Donahue, 2006), animal models (Cook et al., 2006; Elliott et al., 1999), and cadaveric studies (Bedi et al., 2010; Lee et al., 2006; Seitz et al., 2012). For example, finite element modeling of the joint has shown that partial meniscectomy, similar to total meniscectomy, results in decreased contact areas and increased peak stresses in articular cartilage (Peña et al., 2006; Peña et al., 2005; Zielinska and Donahue, 2006). Furthermore, in human cadaveric knees, radial tears or partial meniscectomy significantly increased contact pressures, and surgical repair of the meniscus failed to restore the normal pressure distribution in the knee (Bedi et al., 2012, 2010). However, available in vivo data detailing the changes in tibiofemoral contact mechanics following meniscal tears is limited.

In addition to the altered mechanical environment following a meniscal tear, the biochemical environment may also be affected by joint injury (Brophy et al., 2012; Lindhorst et al., 2000; Lohmander et al., 1999). A variety of biomarkers of cartilage metabolism in the synovial fluid have been associated with the development of osteoarthritis, including matrix metalloproteinases (MMPs) (Baragi et al., 2009; Janusz et al., 2002; Pozgan et al., 2010), sulfated glycosaminoglycans (sGAGs) (Lindhorst et al., 2000; Lohmander et al., 1999), cartilage oligomeric matrix protein (COMP) (Carlson et al., 2002; Wu et al., 2014), prostaglandin $E_{2}$ (PGE2) (Nishimura et al., 2002), and C2C (Fraser et al., 2003), which is a type II collagen neoepitope released upon collagenase cleavage. However, the relationship between catabolic biomarkers in the synovial fluid and cartilage strains following meniscal injury has yet to be studied. Such information could provide insights into the link between biomechanical and biochemical changes in injured joints that may promote the early development of osteoarthritis.

The primary goals of this study were to quantify the effects of meniscal tears on in vivo cartilage strains over a full range of weight bearing flexion angles and to determine the relationship of cartilage strain with biomarkers of cartilage degradation in the synovial fluid. We hypothesize that following a medial meniscus tear, the contact strain in the medial compartment will be
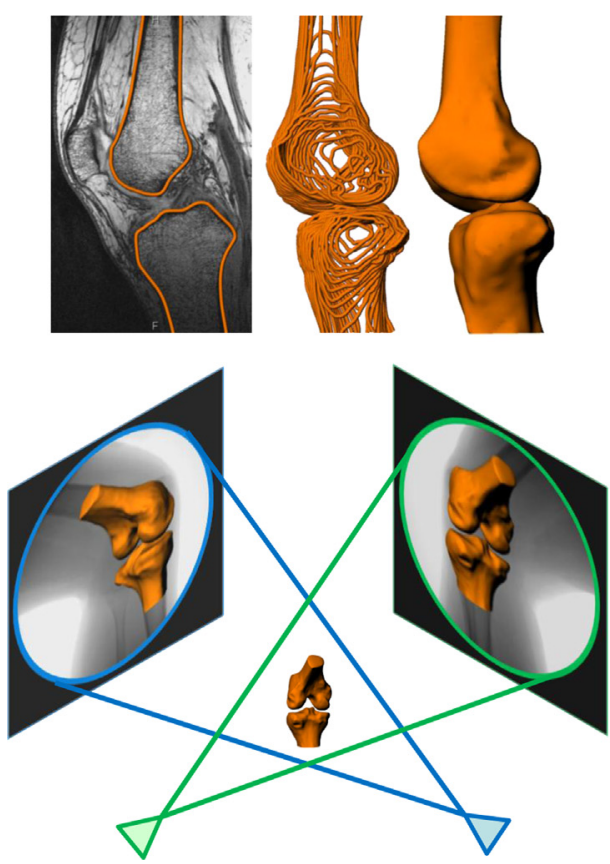

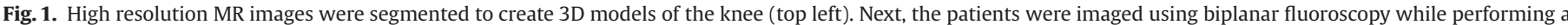
quasi-static lunge (top right). The fluoroscopic images and 3D models were used to reproduce the motion of each knee during the lunge (bottom). 
(Coleman et al., 2013), corresponding to a difference in cartilage thickness of $1 \%$ (Coleman et al., 2013; Widmyer et al., 2013).

\subsection{Fluoroscopic imaging}

Each subject's injured and contralateral uninjured control knee were imaged using biplanar fluoroscopy (Pulsera, Philips, The Netherlands) (Abebe et al., 2011a, b). Digital radiographs were captured while the subject was standing with a single knee positioned between the crossing beams of the fluoroscopes. To evaluate a wide range of motion, each subject performed a quasi-static lunge from full extension to $90^{\circ}$ in increments of $30^{\circ}$. To capture even deeper flexion, we also included a $105^{\circ}$ knee flexion position. Subjects were positioned at each flexion angle using a goniometer. The radiographic images were imported into the solid modeling software to reproduce the position and orientation of the fluoroscopes at the time of testing. For each flexion angle, the MR-based 3D models of the femur and tibia were imported into this system and manually positioned in 6 degrees of freedom until their projections matched their respective silhouette on each of the fluoroscopic images simultaneously (Fig. 1) (DeFrate et al., 2006). Thus, the 3D models were used to reproduce the position of the knee during the lunge sequence (Abebe et al., 2011b; DeFrate et al., 2006). Previous studies have shown that this methodology can reproduce joint motion to within less than $0.1 \mathrm{~mm}$ and $0.3^{\circ}$ (Caputo et al., 2009; DeFrate et al., 2006; Utturkar et al., 2013).

\subsection{Measurement of cartilage strain}

Knowing the relationship of the cartilage surfaces relative to the bones from MRI, areas of overlap of the undeformed tibial and femoral cartilage mesh surfaces were used to calculate tibiofemoral cartilage contact strains in each knee position. Cartilage-to-cartilage contact strains were calculated by taking the ratio of the distance of penetration to the thickness of the two cartilage layers at each point on the tibial cartilage surface (Bischof et al., 2010; Van de Velde et al., 2009) (Fig. 2). Peak strains were calculated on both the injured and uninjured knee in both the medial and lateral compartments at each flexion angle. Differences in peak cartilage contact strains were compared within each compartment using a twoway ANOVA, with flexion angle and the presence or absence of meniscal injury as repeated measures. The Newman-Keuls post hoc test was used to detect differences between means as appropriate. Differences were considered statistically significant at a $95 \%$ confidence level $(p<0.05)$.

\subsection{Synovial fluid biomarkers}

As part of the IRB approved protocol, serum and lavaged synovial fluid was collected at the time of meniscal surgery in seven of the eight subjects. The median time from injury to surgery was 13 weeks and ranged from 4-34 weeks. Synovial fluid was centrifuged for $5 \mathrm{~min}$ at $3500 \times g$ and $4{ }^{\circ} \mathrm{C}$. Then the synovial fluid and serum were aliquoted and stored at $-80{ }^{\circ} \mathrm{C}$ until biomarker analyses were performed.

Total MMP activity was measured as previously published using the quenched fluorogenic substrate Dab-Gly-Pro-Leu-Gly-Met-Arg-Gly-Lys-Flu (Sigma-Aldrich; St. Louis, MO) (McNulty et al., 2009; Wilusz et al., 2008). sGAG content was measured in the synovial fluid using an alcian blue assay (Kamiya Biomedical Company, Seattle, WA). Enzyme-linked immunosorbent assays (ELISAs) were used to measure the following biomarkers in the synovial fluid: COMP (Biovendor, Czech Republic), PGE2 (R \& D Systems, Minneapolis, MN), and C2C (Ibex, Montreal, Canada). Urea measurements were performed on the matched serum and synovial fluid samples using the urea nitrogen reagent set (Pointe Scientific, Canton, MI) and urea standards (CMA Microdialysis, North Chelmsford, MA) to determine the dilution factor for the lavaged synovial fluid. The dilution factor (DF) was calculated as follows: $\mathrm{DF}=($ Serum Urea +0.897$) /$ Synovial Fluid Urea (Kraus et al., 2002). Final concentrations of the biomarkers were corrected for the synovial fluid dilution factor. The synovial fluid measurements were not normally distributed and thus the median values are reported. Spearman correlations were performed to determine the relationship between the average strain in the medial and lateral compartments at $105^{\circ}$ of flexion and the synovial fluid concentrations of each biomarker.

\section{Results}

Peak contact strains in the medial compartment of the injured knee were significantly greater than those of the uninjured knee (Fig. 3, $p=0.008$ ). Across all flexion angles, peak medial compartment strain averaged $17 \pm 5 \%$ (mean \pm standard deviation) in the intact knee, compared to $23 \pm 7 \%$ in the injured knee. No statistically significant effects of flexion angle $(p=0.59)$ or significant interactions between flexion angle and the presence or absence of meniscal injury $(p=0.21)$ were detected.

In the lateral compartment, a statistically significant interaction between flexion angle and meniscal injury was observed (Fig. 4, $p=0.038)$. In the normal knee, the contact strain ranged from $15 \pm 3 \%$ at $0^{\circ}$ of flexion to $17 \pm 3 \%$ at $105^{\circ}$ of flexion. In the injured knee, contact strain was $12 \pm 3 \%$ at $0^{\circ}$ of flexion and increased to $23 \pm 7 \%$ at $105^{\circ}$ of flexion. There was a statistically significant

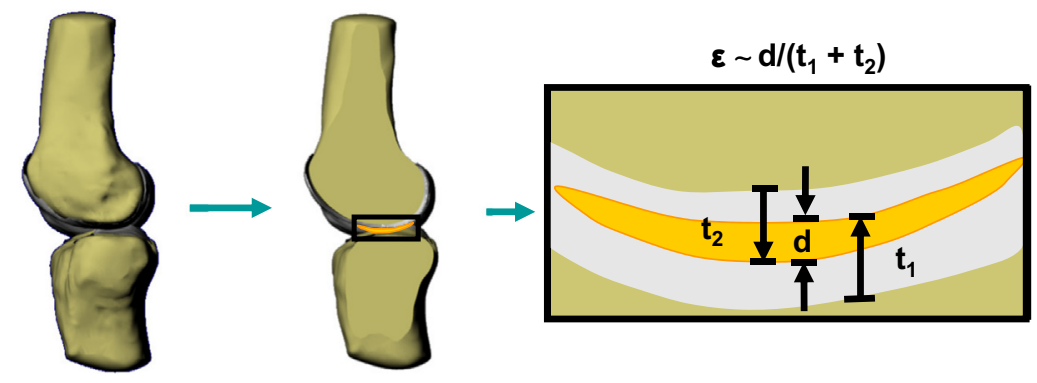

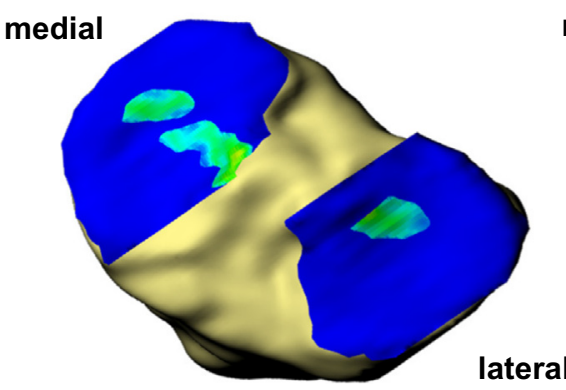

Uninjured medial

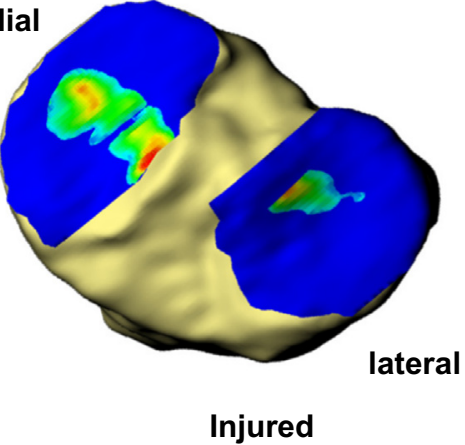

Strain

(\%)
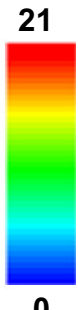

0

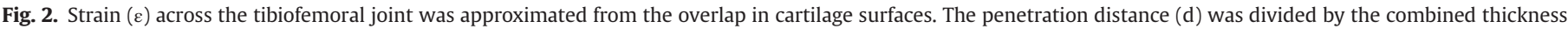

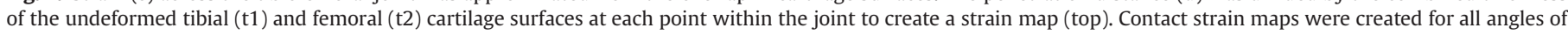

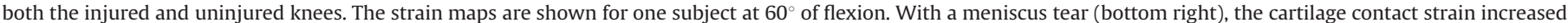
in the medial compartment, as compared to the uninjured knee (bottom left). 


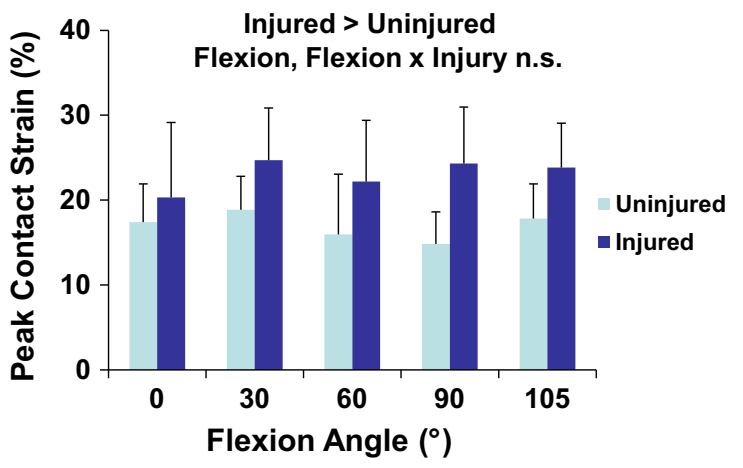

Fig. 3. In the medial compartment, meniscus injury increased peak contact strains. There were no statistically significant effects of flexion or interactions between flexion and injury. Data is presented as the mean peak contact strain+standard deviation.

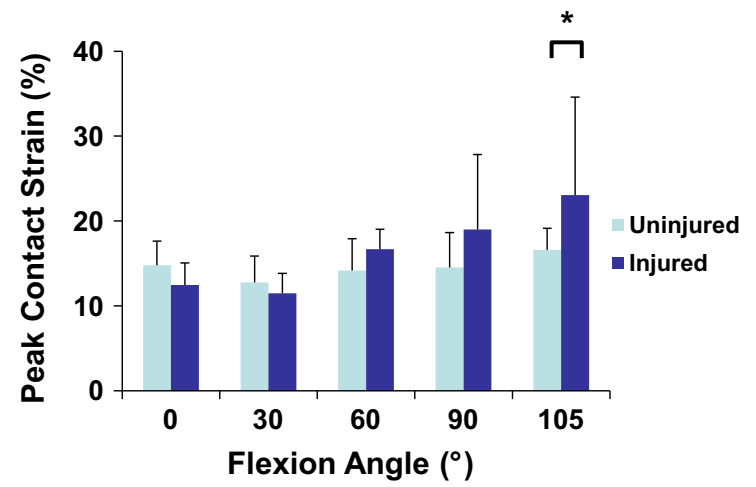

Fig. 4. In the lateral compartment, a statistically significant interaction between flexion angle and meniscal injury was observed. At the maximum flexion angle of $105^{\circ}$, peak contact strains were increased in the injured knee as compared to the contralateral control. Data is presented as the mean peak contact strain+standard deviation.

increase in contact strain between the normal and injured knee at $105^{\circ}$ of flexion $(p=0.029)$.

All of the catabolic biomarkers were detectable in the synovial fluid from the injured knee at the time of joint surgery. The median total MMP activity was $906 \times 10^{3}$ fluorescent units $/ \mathrm{mL}$ in the synovial fluid of the meniscal tear patients (Fig. 5A). The median concentration of each of the other biomarkers in the synovial fluid was $78 \mu \mathrm{g} / \mathrm{mL}$ sGAG (Fig. 5B), $15 \mu \mathrm{g} / \mathrm{mL}$ COMP (Fig. 5C), $25 \mathrm{ng} / \mathrm{mL}$ PGE2 (Fig. 5D), and $69 \mathrm{ng} / \mathrm{mL}$ C2C (Fig. 5E).

Given the significant increases in cartilage strain in both the medial and lateral compartments at $105^{\circ}$ of flexion, we averaged the strain across both compartments to give an average total strain across the tibiofemoral joint. This average strain was then correlated to the concentrations of the biomarkers in the synovial fluid (Table 1). There was a positive correlation between the total MMP activity and average cartilage strain $(R=0.79, p<0.05)$. However, there were no significant correlations between sGAG, COMP, PGE2, or $\mathrm{C} 2 \mathrm{C}$ levels and cartilage strain in these subjects.

\section{Discussion}

This study investigated in vivo cartilage contact strain patterns in knees with injured medial menisci through the use of MRI, biplanar fluoroscopy, and 3D modeling and correlated cartilage strain with biomarkers of cartilage metabolism in the synovial fluid, which have previously been associated with the development of osteoarthritis. Based on the established link of increased risk for early onset osteoarthritis following meniscal tear
(Lohmander et al., 2007), our hypothesis was that there would be increased contact strain in the injured knees and that the catabolic biomarkers would correlate with cartilage strain. In general, our results confirm the hypothesis, demonstrating that medial compartment strains increased significantly across all flexion angles and that lateral compartment strains increased at $105^{\circ}$ of flexion. Overall, the average increase in contact strain following injury was approximately $6 \%$ in the medial compartment. Because peak strains were elevated in both compartments at maximum flexion, correlations were performed between average strain and biomarker levels in this knee position. As a result, we found that average cartilage strain at $105^{\circ}$ of flexion correlated with the total MMP activity in the synovial fluid.

Several previous studies have examined tibiofemoral cartilage contact strains in healthy knees (Bingham et al., 2008) and knees with cruciate ligament deficiencies (Van de Velde et al., 2009). The magnitudes and trends in contact strains reported in this study are consistent with these previous studies (Bingham et al., 2008; Van de Velde et al., 2009). Specifically, these previous studies have shown that strains from the uninjured knee of subjects with ACL injuries and in subjects with healthy knees ranged from $10-30 \%$, with higher values typically occurring in the medial compartment (Bingham et al., 2008; Van de Velde et al., 2009).

The effects of the medial meniscus injury were most evident in the medial compartment, which experienced significantly elevated contact strains compared to the uninjured control knee at all flexion angles. Other studies have investigated the mechanical effects of medial meniscus injuries and treatment on various knee functions in both computational (Bae et al., 2012; Peña et al., 2005; Zielinska and Donahue, 2006) and in vitro (Allaire et al., 2008; Bedi et al., 2010; Lee et al., 2006; Seitz et al., 2012) models. However, these data are typically reported in the form of contact pressures or stresses. While it is not possible to infer contact stresses directly in the current study, the data trends are similar in both model modalities, indicating that loss of medial meniscus integrity results in increases in medial compartment loading. Interestingly, the results of the present study also found increased contact strains in the lateral compartment at $105^{\circ}$ of flexion. Since the medial meniscus is believed to have an important role in transmitting load in higher flexion angles (Lee et al., 2006; Pinskerova et al., 2009; Seitz et al., 2012; Yao et al., 2008), it is likely that disruptions in the position of the knee due to a medial meniscus tear alter load transmission through the lateral compartment as well, as observed in this study.

Altered joint loading is believed to play an important role in the development of osteoarthritis (Guilak, 2011). While the multifactorial origin of mechanically-induced joint degeneration remains to be elucidated, the connection between traumatic knee injury and early osteoarthritis is well established, especially given the high rates of disease progression in those who have ligamentous or meniscal injuries (Lohmander et al., 2007). Because these injuries have been shown to invoke joint kinematic changes during a variety of functions (Ahn et al., 2011; Andriacchi et al., 2004; Bedi et al., 2010, 2012; Chen et al., 2012; Tashman et al., 2004), it has been suggested that these ensuing changes alter cartilage loading detrimentally. Several studies, including our present findings, provide support for this hypothesis by demonstrating alterations in cartilage stress and strain patterns after joint injury (Bedi et al., 2013, 2010; Bischof et al., 2010; Peña et al., 2005; Van de Velde et al., 2009). However, despite the increasing evidence of altered stress and strain distributions in the joint following injury, how these changes relate to mechanical and other physical factors experienced at the cellular and molecular compositional level is less clear (Halloran et al., 2012). In this regard, in vivo strain data, in combination with experimental measures of contact stress (Bedi et al., 2010) and computational 
A

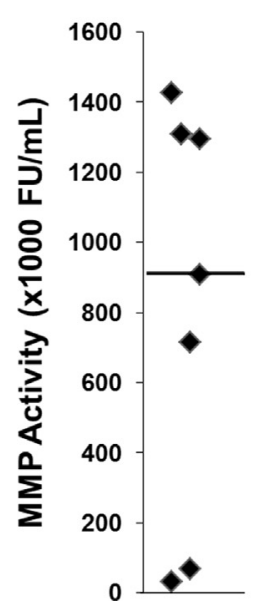

B

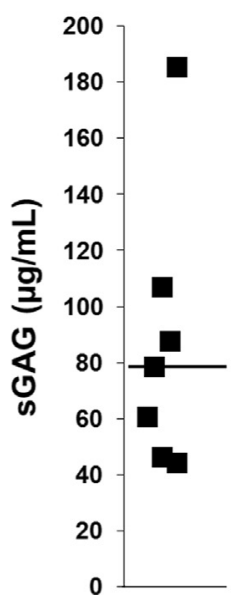

C

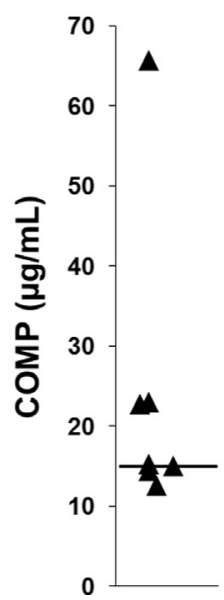

D

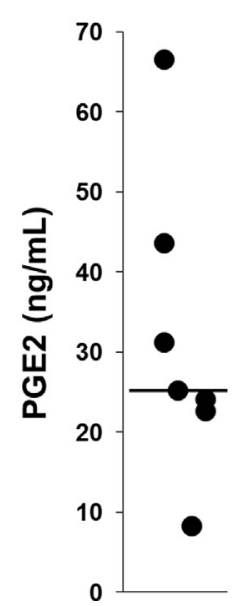

E

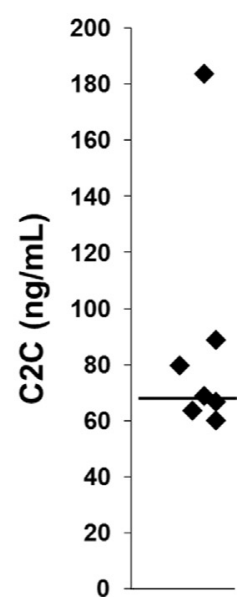

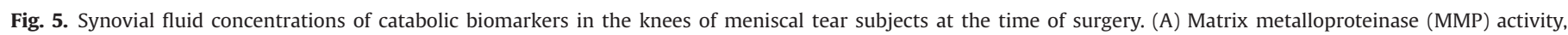

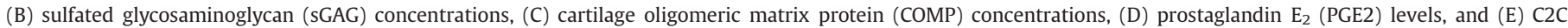
concentrations in the synovial fluid. Each point indicates the concentration for an individual and the median for each biomarker is indicated by the line.

Table 1

Correlation of average cartilage strain to biomarkers.

\begin{tabular}{lcc}
\hline Biomarker & Spearman $\boldsymbol{R}$ & $\boldsymbol{p}$-Value \\
\hline MMP & $\mathbf{0 . 7 9}$ & $\mathbf{0 . 0 4}$ \\
SGAG & -0.64 & 0.12 \\
COMP & -0.57 & 0.18 \\
PGE2 & 0.50 & 0.25 \\
C2C & 0.14 & 0.76 \\
\hline
\end{tabular}

models of the knee (Peña et al., 2006; Wilson et al., 2003; Zielinska and Donahue, 2006) may provide important insights into the mechanobiologic response of cartilage to altered loading (Sanchez-Adams et al., 2014). In this study, we have shown the effects of a meniscal tear on cartilage strain but it is also important to note that the altered mechanical loading in the joint following a meniscal injury likely affects other joint tissues including the meniscus, bone, ligaments, and synovium. In particular, the meniscus, bone, and synovium have been shown to be involved in the production of catabolic biomarkers as well (Brophy et al., 2012; Galliera et al., 2010; Heard et al., 2011).

Cartilage strain following meniscal injury correlated positively with total MMP activity in the synovial fluid. MMPs are a family of enzymes capable of degrading all components of the extracellular matrix, and in particular the collagenases MMP-1, MMP-8, MMP13 , and membrane type 1 MMP (MT1-MMP) are the only enzymes capable of breaking down the intact triple helical collagen (Billinghurst et al., 1997). MMPs have previously been shown to be upregulated following meniscal injury (Brophy et al., 2012; Killian et al., 2011) and to be mechanically regulated (Blain, 2007). In addition, specific inhibition of MMP-13 or broad spectrum MMP inhibition protects against the development of osteoarthritis following medial meniscus tear in a rat model (Baragi et al., 2009; Janusz et al., 2002). Broad spectrum MMP inhibition can also promote integrative meniscal repair in the presence of IL-1 (McNulty et al., 2009). In this study, the total MMP activity measured is a combination of the activity of both the pro-MMPs and active MMPs secreted into the synovial fluid. Very few of the synovial fluid samples contained active MMPs and thus the majority of the MMPs were in the proenzyme form. Therefore, it is possible that active MMPs may be bound in the tissue extracellular matrix or may have degraded during sample processing. Alternatively, the MMPs in the joint are not yet activated. A variety of factors in the joint can activate the pro-MMPs, including plasmin and MT-MMPs (Milner et al., 2001) and also activated protein C (APC), which is elevated in the synovial fluid of osteoarthritic patients (Jackson et al., 2014). Therefore, the measured total MMP activity is a good surrogate for the potential MMP activity in the joint that may contribute to extracellular matrix component breakdown and ultimately osteoarthritis.

The synovial fluid concentrations of SGAG, COMP, PGE2, and $\mathrm{C} 2 \mathrm{C}$ in our study were not correlated with average cartilage strain. Aggrecan turnover is increased following meniscal tears (Lohmander et al., 1999) and with high strain of cartilage and meniscal explants (Killian et al., 2011; Waters et al., 2014). COMP synthesis and release by chondrocytes is mechanically regulated (Piscoya et al., 2005; Wong et al., 1999) and has been shown to be elevated following canine meniscectomy, with peaks around 4-8 weeks after injury (Lindhorst et al., 2000). Previous studies have shown that $30 \mathrm{~min}$ of one-legged knee extension exercise decreases synovial fluid COMP in osteoarthritic knees (Helmark et al., 2012). Furthermore, the knee state and type of exercise impact alterations in COMP levels, as in healthy knees serum COMP levels correlate with cartilage volume changes after drop landing but not after running (Niehoff et al., 2011). PGE2 is also increased in osteoarthritic synovial fluid (Nishimura et al., 2002) and is mechanically regulated in cartilage, as well as meniscus (Fermor et al., 2002; Hennerbichler et al., 2007; Waters et al., 2014). Finally, a trend towards increased C2C was reported at 30 days following a single $8 \mathrm{MPa}$ stress on cartilage explants (Barr et al., 2014). However, in the meniscal tear subjects, longer times following joint injury may be necessary to detect the breakdown of the collagenous matrix.

Since the type of meniscus injury varied between patients and the extent of tissue removed during a meniscectomy varies, future studies may wish to examine changes in joint biomechanics with the loss of different proportions of the meniscus. In cadaveric studies, joint contact stresses increase with the extent of meniscus removal (Baratz et al., 1986; Bedi et al., 2010; Lee et al., 2006). While we did not group our population according to type or severity of medial meniscus tear, the results of our study show that load transmission changes occur after meniscus injury and prior to meniscectomy. Surgical protocols now emphasize maximizing the preservation of the meniscal peripheral rim, in order to minimize changes in contact area and joint stability (Englund, 2008; Lee et al., 2006). Future studies measuring in vivo strain in patients following surgical treatment should provide key information to further revise guidelines on the acceptable limits of 
resection to maintain normal cartilage contact patterns, with the goal of ultimately reducing the risk of osteoarthritis development.

A potential limitation of this study is the relatively small sample size, which was sufficient to determine biomechanical differences and also the strong correlation between strain and MMP activity but is likely limited for the detection of small to modest correlations of other biomarkers. Additionally, the single timepoint of synovial fluid collection at surgery and the wide range of time from injury to collection across the patients may have made correlations difficult to detect. Furthermore, synovial fluid from the contralateral, uninjured knee could provide information on the baseline biomarker profiles of each individual in relation to those of the injured knee. The measured synovial fluid concentrations in the meniscal tear subjects are slightly lower than previously reported for patients following an ACL tear; however, the collection of synovial fluid occurred much closer to the time of injury in the ACL study (average 15 days versus median 13 weeks) (Catterall et al., 2010).

It is important to note that meniscal injuries can occur as a result of an acute trauma or due to tissue degeneration. In general, degenerative tears are associated with increased age and osteoarthritic changes (Berthiaume et al., 2005; Englund, 2008; Greis et al., 2002). Additionally, degenerative tears often have an insidious onset of symptoms without a specific traumatic event initiating injury (Englund, 2008; Howell et al., 2014; Sihvonen et al., 2013). Therefore, to minimize the effects that existing joint degeneration may be playing in the measurements of the present study, patient inclusion criteria were enforced to recruit individuals with an identifiable, traumatic injury and no MRI evidence of cartilage degeneration on the femoral condyles or tibial plateaus of both knees.

In conclusion, this study investigated in vivo tibiofemoral contact strain patterns in patients with an isolated medial meniscus tear and levels of biomarkers of cartilage metabolism in the synovial fluid. Cartilage contact strains increased after meniscal tear when compared to the contralateral control knee, especially at higher flexion angles. In addition, total MMP activity positively correlated with the average cartilage strain across the joint at maximum flexion. These findings suggest that the ability of the meniscus to distribute load is compromised after the injury, thus overloading the cartilage and upregulating mechanically sensitive mediators like the MMPs. These changes likely contribute to the development of osteoarthritis following meniscal injury and support the need to improve surgical treatment options to restore normal cartilage loading.

\section{Conflict of interest statement}

The authors of this manuscript have no conflicts of interest related to the content of the study.

\section{Acknowledgments}

This work was supported in part by the National Institutes of Health grants AR63325, AR65527, AR48182, AR48852, AG15768, AR50245, and AG46927. The technical assistance provided by Dr. Virginia Kraus and Janet Huebner through the Biomarkers Shared Resource at Duke University Medical Center is also gratefully acknowledged.

\section{References}

Abebe, E.S., Kim, J.P., Utturkar, G.M., Taylor, D.C., Spritzer, C.E., Moorman 3rd, C.T., Garrett, W.E., DeFrate, L.E., 2011a. The effect of femoral tunnel placement on ACL graft orientation and length during in vivo knee flexion. J. Biomech. 44, 1914-1920.
Abebe, E.S., Utturkar, G.M., Taylor, D.C., Spritzer, C.E., Kim, J.P., Moorman 3rd, C.T. Garrett, W.E., DeFrate, L.E., 2011b. The effects of femoral graft placement on in vivo knee kinematics after anterior cruciate ligament reconstruction. J. Biomech. 44, 924-929.

Abrams, G.D., Frank, R.M., Gupta, A.K., Harris, J.D., McCormick, F.M., Cole, B.J., 2013 Trends in meniscus repair and meniscectomy in the United States, 2005-2011. Am. J. Sports Med. 41, 2333-2339.

Ahmed, A.M., Burke, D.L., 1983. In-vitro measurement of static pressure distribution in synovial joints-Part I: Tibial surface of the knee. J. Biomech. Eng. 105, 216-225.

Ahn, J.H., Bae, T.S., Kang, K.S., Kang, S.Y., Lee, S.H., 2011. Longitudinal tear of the medial meniscus posterior horn in the anterior cruciate ligament-deficient knee significantly influences anterior stability. Am. J. Sports Med. 39, 2187-2193.

Allaire, R., Muriuki, M., Gilbertson, L., Harner, C.D., 2008. Biomechanical consequences of a tear of the posterior root of the medial meniscus. Similar to total meniscectomy. J. Bone Joint Surg. Am. 90, 1922-1931.

Andersson-Molina, H., Karlsson, H., Rockborn, P., 2002. Arthroscopic partial and total meniscectomy: a long-term follow-up study with matched controls. Arthroscopy 18, 183-189.

Andriacchi, T.P., Mundermann, A., Smith, R.L., Alexander, E.J., Dyrby, C.O., Koo, S. 2004. A framework for the in vivo pathomechanics of osteoarthritis at the knee. Ann. Biomed. Eng. 32, 447-457.

Arendt, E.A., American Orthopaedic Society for Sports Medicine, American Academy of Orthopaedic Surgeons, 1999. OKU Orthopaedic Knowledge Update. Sports Medicine 2, 1st ed. American Academy of Orthopaedic Surgeons, Rosemont, IL.

Badlani, J.T., Borrero, C., Golla, S., Harner, C.D., Irrgang, J.J., 2013. The effects of meniscus injury on the development of knee osteoarthritis: data from the osteoarthritis initiative. Am. J. Sports Med. 41, 1238-1244.

Bae, J.Y., Park, K.S., Seon, J.K., Kwak, D.S., Jeon, I., Song, E.K., 2012. Biomechanical analysis of the effects of medial meniscectomy on degenerative osteoarthritis. Med. Biol. Eng. Comput. 50, 53-60.

Baker, B.S., Lubowitz, J., 2006. Meniscus Injuries WebMD. In: Milne, L., Talavera, F. Goitz, H.T., Whitehurst, J.B., Young, C.C. (Eds.), Emedicine.

Baragi, V.M., Becher, G., Bendele, A.M., Biesinger, R., Bluhm, H., Boer, J., Deng, H. Dodd, R., Essers, M., Feuerstein, T., Gallagher Jr., B.M., Gege, C., Hochgurtel, M., Hofmann, M., Jaworski, A., Jin, L., Kiely, A., Korniski, B., Kroth, H., Nix, D., Nolte B., Piecha, D., Powers, T.S., Richter, F., Schneider, M., Steeneck, C., Sucholeiki, I., Taveras, A., Timmermann, A., Van Veldhuizen, J., Weik, J., Wu, X., Xia, B., 2009. A new class of potent matrix metalloproteinase 13 inhibitors for potential treatment of osteoarthritis: Evidence of histologic and clinical efficacy without musculoskeletal toxicity in rat models. Arthritis Rheum. 60, 2008-2018.

Baratz, M.E., Fu, F.H., Mengato, R., 1986. Meniscal tears: the effect of meniscectomy and of repair on intraarticular contact areas and stress in the human knee. A preliminary report. Am. J. Sports Med. 14, 270-275.

Barr, L., Getgood, A., Guehring, H., Rushton, N., Henson, F.M., 2014. The effect of recombinant human fibroblast growth factor-18 on articular cartilage following single impact load. J. Orthop. Res. 32, 923-927.

Bedi, A., Chen, T., Santner, T.J., El-Amin, S., Kelly, N.H., Warren, R.F., Maher, S.A. 2013. Changes in dynamic medial tibiofemoral contact mechanics and kinematics after injury of the anterior cruciate ligament: a cadaveric model. Proc Inst. Mech. Eng., H 227, 1027-1037.

Bedi, A., Kelly, N., Baad, M., Fox, A J. Ma, Y., Warren, R.F., Maher S. A. 2012 . Dynamic contact mechanics of radial tears of the lateral meniscus: implications for treatment. Arthroscopy 28, 372-381.

Bedi, A., Kelly, N.H., Baad, M., Fox, A.J., Brophy, R.H., Warren, R.F., Maher, S.A., 2010 Dynamic contact mechanics of the medial meniscus as a function of radial tear, repair, and partial meniscectomy. J. Bone Joint Surg. Am. 92, 1398-1408.

Berthiaume, M.-J., Raynauld, J.-P., Martel-Pelletier, J., Labonté, F., Beaudoin, G., Bloch, D.A., Choquette, D., Haraoui, B., Altman, R.D., Hochberg, M., Meyer, J.M., Cline, G.A., Pelletier, J.-P., 2005. Meniscal tear and extrusion are strongly associated with progression of symptomatic knee osteoarthritis as assessed by quantitative magnetic resonance imaging. Ann. Rheum. Dis. 64, 556-563.

Billinghurst, R.C., Dahlberg, L., Ionescu, M., Reiner, A., Bourne, R., Rorabeck, C., Mitchell, P., Hambor, J., Diekmann, O., Tschesche, H., Chen, J., Van Wart, H. Poole, A.R., 1997. Enhanced cleavage of type II collagen by collagenases in osteoarthritic articular cartilage. J. Clin. Invest. 99, 1534-1545.

Bingham, J.T., Papannagari, R., Van de Velde, S.K., Gross, C., Gill, T.J., Felson, D.T. Rubash, H.E., Li, G., 2008. In vivo cartilage contact deformation in the healthy human tibiofemoral joint. Rheumatology 47, 1622-1627.

Bischof, J.E., Spritzer, C.E., Caputo, A.M., Easley, M.E., DeOrio, J.K., Nunley 2nd, J.A., DeFrate, L.E., 2010. In vivo cartilage contact strains in patients with lateral ankle instability. J. Biomech. 43, 2561-2566.

Blain, E.J., 2007. Mechanical regulation of matrix metalloproteinases. Front. Biosci. 12, 507-527.

Brophy, R.H., Rai, M.F., Zhang, Z., Torgomyan, A., Sandell, L.J., 2012. Molecular analysis of age and sex-related gene expression in meniscal tears with and without a concomitant anterior cruciate ligament tear. J. Bone Joint Surg. Am. 94, 385-393.

Caputo, A.M., Lee, J.Y., Spritzer, C.E., Easley, M.E., DeOrio, J.K., Nunley 2nd, J.A. DeFrate, L.E., 2009. In vivo kinematics of the tibiotalar joint after lateral ankle instability. Am. J. Sports Med. 37, 2241-2248.

Carlson, C.S., Guilak, F., Vail, T.P., Gardin, J.F., Kraus, V.B., 2002. Synovial fluid biomarker levels predict articular cartilage damage following complete medial meniscectomy in the canine knee. J. Orthop. Res. 20, 92-100. 
Catterall, J.B., Stabler, T.V., Flannery, C.R., Kraus, V.B., 2010. Changes in serum and synovial fluid biomarkers after acute injury (NCT00332254). Arthritis Res. Ther. 12, R229.

Chen, C.H., Li, J.S., Hosseini, A., Gadikota, H.R., Gill, T.J., Li, G., 2012. Anteroposterior stability of the knee during the stance phase of gait after anterior cruciate ligament deficiency. Gait Posture 35, 467-471.

Christoforakis, J., Pradhan, R., Sanchez-Ballester, J., Hunt, N., Strachan, R.K., 2005. Is there an association between articular cartilage changes and degenerative meniscus tears? the Journal of Arthroscopic and Related Surgery. Arthroscopy 21, 1366-1369.

Coleman, J.L., Widmyer, M.R., Leddy, H.A., Utturkar, G.M., Spritzer, C.E., Moorman 3rd, C.T., Guilak, F., DeFrate, L.E., 2013. Diurnal variations in articular cartilage thickness and strain in the human knee. J. Biomech. 46, 541-547.

Cook, J.L., Fox, D.B., Malaviya, P., Tomlinson, J.L., Kuroki, K., Cook, C.R., Kladakis, S. 2006. Long-term outcome for large meniscal defects treated with small intestinal submucosa in a dog model. Am. J. Sports Med. 34, 32-42.

DeFrate, L.E., Papannagari, R., Gill, T.J., Moses, J.M., Pathare, N.P., Li, G., 2006. The 6 degrees of freedom kinematics of the knee after anterior cruciate ligament deficiency. Am. J. Sports Med. 34, 1240-1246.

Elliott, D.M., Guilak, F., Vail, T.P., Wang, J.Y., Setton, L.A., 1999. Tensile properties of articular cartilage are altered by meniscectomy in a canine model of osteoarthritis. J. Orthop. Res. 17, 503-508.

Englund, M., 2008. The role of the meniscus in osteoarthritis genesis. Rheum. Dis Clin. North Am. 34, 573-579.

Fauno, P., Nielsen, A.B., 1992. Arthroscopic partial meniscectomy: a long-term follow-up. Arthroscopy 8, 345-349.

Fermor, B., Weinberg, J.B., Pisetsky, D.S., Misukonis, M.A., Fink, C., Guilak, F., 2002 Induction of cyclooxygenase-2 by mechanical stress through a nitric oxideregulated pathway. Osteoarthritis Cartilage 10, 792-798.

Fraser, A., Fearon, U., Billinghurst, R.C., Ionescu, M., Reece, R., Barwick, T., Emery, P., Poole, A.R., Veale, D.J., 2003. Turnover of type II collagen and aggrecan in cartilage matrix at the onset of inflammatory arthritis in humans: relationship to mediators of systemic and local inflammation. Arthritis Rheum. 48, 3085-3095.

Galliera, E., Randelli, P., Dogliotti, G., Dozio, E., Colombini, A., Lombardi, G., Cabitza, P., Corsi, M.M., 2010. Matrix metalloproteases MMP-2 and MMP-9: are they early biomarkers of bone remodelling and healing after arthroscopic acromioplasty? Injury 41, 1204-1207.

Greis, P.E., Bardana, D.D., Holmstrom, M.C., Burks, R.T., 2002. Meniscal injury: Basic science and evaluation. J. Am. Acad. Orthop. Surg. 10, 168-176.

Guilak, F., 2011. Biomechanical factors in osteoarthritis. Best Pract. Res. Clin. Rheumatol. 25, 815-823.

Hall, M., Wrigley, T.V., Metcalf, B.R., Cicuttini, F.M., Wang, Y., Hinman, R.S., Dempsey A.R., Mills, P.M., Lloyd, D.G., Bennell, K.L., 2014. Do moments and strength predict cartilage changes following partial meniscectomy? Med. Sci. Sports Exerc. [Epub ahead of print]

Halloran, J.P., Sibole, S., van Donkelaar, C.C., van Turnhout, M.C., Oomens, C.W. Weiss, J.A., Guilak, F., Erdemir, A., 2012. Multiscale mechanics of articula cartilage: potentials and challenges of coupling musculoskeletal, joint, and microscale computational models. Ann. Biomed. Eng. 40, 2456-2474.

Haut Donahue, T.L., Hull, M.L., Rashid, M.M., Jacobs, C.R., 2004. The sensitivity of tibiofemoral contact pressure to the size and shape of the lateral and medial menisci. J. Orthop. Res. 22, 807-814.

Heard, B.J., Achari, Y., Chung, M., Shrive, N.G., Frank, C.B., 2011. Early joint tissue changes are highly correlated with a set of inflammatory and degradative synovial biomarkers after ACL autograft and its sham surgery in an ovine model. J. Orthop. Res. 29, 1185-1192.

Helmark, I.C., Petersen, M.C., Christensen, H.E., Kjaer, M., Langberg, H., 2012 Moderate loading of the human osteoarthritic knee joint leads to lowering of intraarticular cartilage oligomeric matrix protein. Rheumatol. Int. 32 1009-1014.

Hennerbichler, A., Fermor, B., Hennerbichler, D., Weinberg, J.B., Guilak, F., 2007 Regional differences in prostaglandin E2 and nitric oxide production in the knee meniscus in response to dynamic compression. Biochem. Biophys. Res. Commun. 358, 1047-1053.

Hoser, C., Fink, C., Brown, C., Reichkendler, M., Hackl, W., Bartlett, J., 2001. Longterm results of arthroscopic partial lateral meniscectomy in knees without associated damage. J. Bone Joint Surg. Br. 83-B, 513-516.

Howell, R., Kumar, N.S., Patel, N., Tom, J., 2014. Degenerative meniscus: pathogenesis, diagnosis, and treatment options. World J. Orthop. 5, 597-602.

Hunter, D.J., Zhang, Y.O., Niu, J.B., Tu, X., Amin, S., Clancy, M., Guermazi, A., Grigorian, M., Gale, D., Felson, D.T., 2006. The association of meniscal pathologic changes with cartilage loss in symptomatic knee osteoarthritis. Arthritis Rheum. 54, 795-801.

Hutchinson, I.D., Moran, C.J., Potter, H.G., Warren, R.F., Rodeo, S.A., 2014, Restoration of the meniscus: form and function. Am. J. Sports Med. 42, 987-998.

Jackson, M.T., Moradi, B., Smith, M.M., Jackson, C.J., Little, C.B., 2014. Activation of matrix metalloproteinases 2,9 , and 13 by activated protein $C$ in human osteoarthritic cartilage chondrocytes. Arthritis Rheumatol. 66, 1525-1536.

Janusz, M.J., Bendele, A.M., Brown, K.K., Taiwo, Y.O., Hsieh, L., Heitmeyer, S.A., 2002. Induction of osteoarthritis in the rat by surgical tear of the meniscus: inhibition of joint damage by a matrix metalloproteinase inhibitor. Osteoarthritis Cartilage 10, 785-791.

Katz, J.N., Meredith, D.S., Lang, P., Creel, A.H., Yoshioka, H., Neumann, G., Fossel, A.H., de Pablo, P., Losina, E., 2006. Associations among preoperative MRI features and functional status following arthroscopic partial meniscectomy. Osteoarthritis Cartilage 14, 418-422.

Killian, M.L., Zielinska, B., Gupta, T., Haut Donahue, T.L., 2011. In vitro inhibition of compression-induced catabolic gene expression in meniscal explants following treatment with IL-1 receptor antagonist. J. Orthop. Sci. 16, 212-220.

Kraus, V.B., Huebner, J.L., Fink, C., King, J.B., Brown, S., Vail, T.P., Guilak, F., 2002. Urea as a passive transport marker for arthritis biomarker studies. Arthritis Rheum. 46, 420-427.

Lee, S.J., Aadalen, K.J., Malaviya, P., Lorenz, E.P., Hayden, J.K., Farr, J., Kang, R.W., Cole, B.J., 2006. Tibiofemoral contact mechanics after serial medial meniscectomies in the human cadaveric knee. Am. J. Sports Med. 34, 1334-1344.

Lindhorst, E., Vail, T.P., Guilak, F., Wang, H., Setton, L.A., Vilim, V., Kraus, V.B., 2000. Longitudinal characterization of synovial fluid biomarkers in the canine meniscectomy model of osteoarthritis. J. Orthop. Res. 18, 269-280.

Lohmander, L.S., Englund, P.M., Dahl, L.L., Roos, E.M., 2007. The long-term consequence of anterior cruciate ligament and meniscus injuries: osteoarthritis. Am. J. Sports Med. 35, 1756-1769.

Lohmander, L.S., Ionescu, M., Jugessur, H., Poole, A.R., 1999. Changes in joint cartilage aggrecan after knee injury and in osteoarthritis. Arthritis Rheum. 42, 534-544.

Lohmander, L.S., Roos, H., Dahlberg, L., Hoerrner, L.A., Lark, M.W., 1994. Temporal patterns of stromelysin-1, tissue inhibitor, and proteoglycan fragments in human knee joint fluid after injury to the cruciate ligament or meniscus. J. Orthop. Res. 12, 21-28.

Maffulli, N., Longo, U.G., Campi, S., Denaro, V., 2010. Meniscal tears. Open Access J. Sports Med. 1, 45-54.

Majewski, M., Susanne, H., Klaus, S., 2006. Epidemiology of athletic knee injuries: a 10-year study. Knee 13, 184-188.

Markolf, K.L., Bargar, W.L., Shoemaker, S.C., Amstutz, H.C., 1981. The role of joint load in knee stability. J. Bone Joint Surg. Am. 63, 570-585.

McNulty, A.L., Weinberg, J.B., Guilak, F., 2009. Inhibition of matrix metalloproteinases enhances in vitro repair of the meniscus. Clin. Orthop. Relat. Res. 467, 1557-1567.

Milner, J.M., Elliott, S.-F., Cawston, T.E., 2001. Activation of procollagenases is a key control point in cartilage collagen degradation: interaction of serine and metalloproteinase pathways. Arthritis Rheum. 44, 2084-2096.

Niehoff, A., Muller, M., Bruggemann, L., Savage, T., Zaucke, F., Eckstein, F., MullerLung, U., Bruggemann, G.P., 2011. Deformational behaviour of knee cartilage and changes in serum cartilage oligomeric matrix protein (COMP) after running and drop landing. Osteoarthritis Cartilage 19, 1003-1010.

Nishimura, M., Segami, N., Kaneyama, K., Suzuki, T., Miyamaru, M., 2002. Relationships between pain-related mediators and both synovitis and joint pain in patients with internal derangements and osteoarthritis of the temporomandibular joint. Oral Surg. Oral Med. Oral Pathol. Oral Radiol. Endod. 94, 328-332.

Peña, E Calvo, B. Martínez, M.A., Palanca, D., Doblaré, M., 2006. Why latera meniscectomy is more dangerous than medial meniscectomy. A finite element study. J. Orthop. Res. 24, 1001-1010.

Peña, E., Calvo, B. Martínez, M.A., Palanca, D., Doblaré, M., 2005, Finite element analysis of the effect of meniscal tears and meniscectomies on human knee biomechanics. Clin. Biomech. 20, 498-507.

Pinskerova, V., Samuelson, K.M., Stammers, J., Maruthainar, K., Sosna, A., Freeman, M.A., 2009. The knee in full flexion: an anatomical study. J. Bone Joint Surg. Br. $91,830-834$.

Piscoya, J.L., Fermor, B., Kraus, V.B., Stabler, T.V., Guilak, F., 2005. The influence of mechanical compression on the induction of osteoarthritis-related biomarkers in articular cartilage explants. Osteoarthritis Cartilage 13, 1092-1099.

Pozgan, U., Caglic, D., Rozman, B., Nagase, H., Turk, V., Turk, B., 2010. Expression and activity profiling of selected cysteine cathepsins and matrix metalloproteinases in synovial fluids from patients with rheumatoid arthritis and osteoarthritis. Biol. Chem. 391, 571-579.

Rangger, C., Klestil, T., Gloetzer, W., Kemmler, G., Benedetto, K.P., 1995. Osteoarthritis After Arthroscopic Partial Meniscectomy. Am. J. Sports Med. 23, $240-244$.

Roos, H., Lauren, M., Adalberth, T., Roos, E.M., Jonsson, K., Lohmander, L.S., 1998. Knee osteoarthritis after meniscectomy: prevalence of radiographic changes after twenty-one years, compared with matched controls. Arthritis Rheum. 41, 687-693.

Sanchez-Adams, J., Leddy, H.A., McNulty, A.L., O'Conor, C.J., Guilak, F., 2014. The mechanobiology of articular cartilage: bearing the burden of osteoarthritis. Curr. Rheumatol. Rep. 16, 451.

Seitz, A.M., Lubomierski, A., Friemert, B., Ignatius, A., Dürselen, L., 2012. Effect of partial meniscectomy at the medial posterior horn on tibiofemoral contact mechanics and meniscal hoop strains in human knees. J. Orthop. Res. 30 (6), 934-942. http://dx.doi.org/10.1002/jor.22010 (Epub November 9, 2011).

Sharma, L., Eckstein, F., Song, J., Guermazi, A., Prasad, P., Kapoor, D., Cahue, S., Marshall, M., Hudelmaier, M., Dunlop, D., 2008. Relationship of meniscal damage, meniscal extrusion, malalignment, and joint laxity to subsequent cartilage loss in osteoarthritic knees. Arthritis Rheum. 58, 1716-1726.

Sihvonen, R., Paavola, M., Malmivaara, A., Itala, A., Joukainen, A., Nurmi, H., Kalske, J., Jarvinen, T.L., Finnish Degenerative Meniscal Lesion Study, G., 2013. Arthroscopic partial meniscectomy versus sham surgery for a degenerative meniscal tear. N. Engl. J. Med. 369, 2515-2524.

Tashman, S., Anderst, W., Kolowich, P., Havstad, S., Arnoczky, S., 2004. Kinematics of the ACL-deficient canine knee during gait: serial changes over two years. J. Orthop. Res. 22, 931-941. 
Utturkar, G.M., Irribarra, L.A., Taylor, K.A., Spritzer, C.E., Taylor, D.C., Garrett, W.E., Defrate, L.E., 2013. The effects of a valgus collapse knee position on in vivo ACL elongation. Ann. Biomed. Eng. 41, 123-130.

Van de Velde, S.K., Bingham, J.T., Hosseini, A., Kozanek, M., DeFrate, L.E., Gill, T.J., Li, G., 2009. Increased tibiofemoral cartilage contact deformation in patients with anterior cruciate ligament deficiency. Arthritis Rheum. 60, 3693-3702.

Waters, N.P., Stoker, A.M., Carson, W.L., Pfeiffer, F.M., Cook, J.L., 2014. Biomarkers affected by impact velocity and maximum strain of cartilage during injury. J. Biomech. 47, 3185-3195.

Widmyer, M.R., Utturkar, G.M., Leddy, H.A., Coleman, J.L., Spritzer, C.E., Moorman 3rd, C.T., DeFrate, L.E., Guilak, F., 2013. High body mass index is associated with increased diurnal strains in the articular cartilage of the knee. Arthritis Rheum. 65, 2615-2622.

Wilson, W., van Rietbergen, B., van Donkelaar, C.C., Huiskes, R., 2003. Pathways of load-induced cartilage damage causing cartilage degeneration in the knee after meniscectomy. J. Biomech. 36, 845-851.

Wilusz, R.E., Weinberg, J.B., Guilak, F., McNulty, A.L., 2008. Inhibition of integrative repair of the meniscus following acute exposure to interleukin-1 in vitro. J. Orthop. Res. 26, 504-512.
Wojtys, E.M., Chan, D.B., 2005. Meniscus structure and function. AAOS Instr. Course Lect. 54, 323-330.

Wong, M., Siegrist, M., Cao, X., 1999. Cyclic compression of articular cartilage explants is associated with progressive consolidation and altered expression pattern of extracellular matrix proteins. Matrix Biol. 18, 391-399.

Wu, P., Holguin, N., Silva, M.J., Fu, M., Liao, W., Sandell, L.J., 2014. Early response of mouse joint tissue to noninvasive knee injury suggests treatment targets. Arthritis Rheumatol. 66, 1256-1265.

Wyland, D.J., Guilak, F., Elliott, D.M., Setton, L.A., Vail, T.P., 2002. Chondropathy after meniscal tear or partial meniscectomy in a canine model. J. Orthop. Res. 20 996-1002.

Yao, J., Lancianese, S.L., Hovinga, K.R., Lee, J., Lerner, A.L., 2008. Magnetic resonance image analysis of meniscal translation and tibio-menisco-femoral contact in deep knee flexion. J. Orthop. Res. 26, 673-684.

Zielinska, B., Donahue, T.L., 2006. 3D finite element model of meniscectomy: changes in joint contact behavior. J. Biomech. Eng. 128, 115-123. 